Background Road traffic injuries have reached epidemic proportion in Sri Lanka with rapid urbanisation and motorization. Due to inadequate consideration, adolescents are forced to share their transportation and recreational space with vehicles. The objective of this study is to assess the built-environment around schools in Sri Lanka.

Methods An observatory study was conducted around 16 high schools in Galle, Sri Lanka. Researchers observed the road conditions and road facilities, and measured the density of vehicles and pedestrians during the school rush hour. A cross-sectional survey was conducted among adolescents from these 16 schools, through which students self-reported their experience of road traffic crashes in the past 6 months. Descriptive analysis and regression model were performed by using STATA. The study was approved by IRB at Duke University and Ruhuna University. Results The built-environment observation showed that although over $80 \%$ of roads around 16 schools were fully paved, $62.5 \%$ roads were narrow due to high pedestrian density and parked vehicles. $18.8 \%$ of roads were one-way road and $37.5 \%$ of roads didn't have clear directions and lanes. Only 18.8\% roads had formal road shoulders to separate pedestrians from vehicles. $56.3 \%$ of schools had a policeman in front of the school gate to control the traffic, but none of the roads had a traffic light to control the vehicles and pedestrians. The regression model showed that after control the school and gender, one-way road (OR: 0.80, 95\% CI: $0.68,0.95)$ and having curve (OR: $0.79,95 \%$ CI: 0.71, 0.89) are protective factors that significantly associated with adolescents' involvement of the crashes.

Conclusions The current built environment around schools in Galle, Sri Lanka is poor. A comprehensive strategy including improving the built environment with the consideration of vulnerable road users is promising to protect adolescents from road traffic injuries.

\section{Safe Communities}

\section{Post Mon 1.21}

\section{ENHANCING VOLUNTEER TRAFFIC POLICE .TO PREVENT PROBLEMS OF ROAD ACCIDENTS IN THE COMMUNITY}

Polcol Arnon Namprasert. Nong Bua Lam Phu Province (NBLP) THAILAND

\subsection{6/injuryprev-2016-042156.616}

Background In 2012 NBLP community dead 86 people and 5,782 were injured from traffic accidents. Most incidents (70\%) occurred at the community roads and motorcycle were the major causes. The victims were mostly young people who were the main worker of the families. They were mostly careless in driving; drunk driving, motorcycling without helmet and speed driving. In addition, there were insufficient officers to regulate manage traffic systems in the community

Solving measures to enhance people participation in traffic management by developing local traffic rules and set up volunteer patrol in the community in order to promote safe driving behaviours in the community. Objectives 1 .To enhance people participation to help solve problems of traffic accidents in the community 2. To develop safe driving culture in the community in order to reduce injuries and deaths from traffic accidents.

Operations The local police authorities discuss with community leaders in order to set local driving rules such as speed limit, no drunken drivers and putting on helmets all the time for motorcycles. They also investigate black spots. Most importantly, recruit volunteer for traffic patrol and control training. This includes learning about traffic rules, traffic signals, the use of alcohol checking equipments and technique on checking on road vehicles. After training, all volunteers had been appointed by local police stations to take part in traffic regulating activities at the check points as well as patrolling. It is important to note that the traffic regulating operation has to be compiled to community rules and cultures

Outputs In 2012, NBLP provinces has developed over 800 volunteers who could take part in traffic management. The measures had solved the problems of inadequate police staffs to manage local traffic systems. As a results, the local traffic accidents reduced by $60 \%$ and people have become aware of the problems and willingly to participate in the agreed rules and regulation measures.

Outcomes Major achievements were the increase in people awareness and participation. The operations had been accepted by local government who now sponsored the volunteer training program and continue integrated road traffic prevention programs with other agencies in NBLP. Moreover, the training and the development traffic volunteer model has been adopted in many provinces. at present, there are more than 20,000 volunteers traffic police in Thailand.

\section{ADVOCACY OF COMMUNITY ACCIDENT PREVENTION (COMMUNITY TRAFFIC SAFETY CHECKPOINT) DURING SONGKRAN FESTIVAL IN THAILAND , 2015}

Natthakarn Waiyanate. Bureau of Non Communicable Disease, Department of Disease Control and Nation Multidisciplinary Road Safety Team Prevention Team, Thailand

\subsection{6/injuryprev-2016-042156.617}

Abstract This study aims at examining the factors causing traffic accident at the community level and finding measures for accident prevention and accident-related risk behaviour reduction to ensure safety of people in the community. At the community level, the accident prevention consists of finding risk group, risk area, establishing community traffic safety checkpoint which is to stop and reduce risk behaviour. The data was collected by Questionnaires and observation of traffic-related risk behaviour among car and motorcycle users passing through the checkpoints in Surat Thani, Chiangmai, Phrae, Chiangrai, and Konkean during Songkran Festival in 2015. The result shows that during 9-15 April 2015 of Songkran Festival (data from 10 provinces, 22 districts), there were 121 injuries, 6 of them had to be admitted to the hospital and there was no death.

Compared to 2014 , death reduces by 5 , injury reduces by 553 , and number of crash victims who were admitted to the hospital reduces by 20 . Also, it is found that traffic accidents heavily occurred on 12-14 April. From Alcohol breath test results, it is highlighted that alcohol level of drivers is especially high during 9-13 April. With regard to helmet wearing behaviour, the community traffic safety checkpoints used various measures to reduce helmet non-wearing behaviour among motorcycle riders who didn't wear helmet such as lending a helmet to them and prohibiting them from passing through the checkpoint unless they came back wearing a helmet. Community traffic safety checkpoints were operated by following 3 . The evaluation underlines that there was preparation of community accident prevention (97.90\%); there were community checkpoints to stop risk group and reduce accidents (100\%); and there was monitoring for 
improvement and success (96.60\%). The province that is most ready for the community traffic safety checkpoint operation is Burirum. A pilot community with full capacity of community traffic safety checkpoint is more ready to operate than a pilot community with partial capacity. Number of accidents in a pilot community with full capacity of community traffic safety checkpoint is less than a pilot community with partial capacity. No accident occurred in a pilot community with full capacity of community traffic safety checkpoint which is highly ready for accident prevention activities.

\section{BUILDING THE CONVERSATION FOR HOSPITAL-BASED INJURY PREVENTION PROGRAM CAPACITY IN TEXAS, USA}

${ }^{1}$ Mary Ann Contreras, ${ }^{2}$ Courtney Edwards, ${ }^{3}$ Shelli Stephens-Stidham, ${ }^{4}$ Stewart Williams. ${ }^{1}$ JPS Health System, Fort Worth, Texas, USA; ${ }^{2}$ Parkland Health and Hospital System, Dallas, Texas, USA; ${ }^{3}$ Parkland Health and Hospital System, Dallas, Texas, USA; ${ }^{4}$ Dell Children's Medical Centre, Austin, Texas

\subsection{6/injuryprev-2016-042156.618}

Background Trauma centres in the U.S. seeking verification by the American College of Surgeons must meet certain criteria outlined in 2014 Resources for Optimal Care of the Injured Patient. The criteria state that trauma centres have an "organised and effective approach to injury prevention."

Description of the problem There are well-defined guidelines for state and local public health injury programs; however, guidance for hospital-based programs has been general and left to individual interpretation. Several U.S. trauma/injury prevention associations and organisations have expressed an interest in developing stronger guidelines for hospital-based injury prevention programs. In 2014, the Texas Governor's EMS and Trauma Advisory Council (GETAC) Injury Prevention Committee convened a work group of Texas representatives from trauma-related associations to reach consensus on standard hospital-based injury prevention program core components for Texas hospitals.

Results Standardised components were developed with input from representatives of the invited Texas organisations, including representatives from all levels of trauma designation (I-IV). Resource documents from U.S. organisations were utilised. The components were developed to be applicable to all hospital-based injury programs regardless of staffing levels or capacity.

A document, Hospital-Based Injury Prevention Components, was developed to explain and provide supporting materials for each identified core component. The document was endorsed by GETAC in November 2014 and disseminated to Texas hospital trauma and injury prevention professionals in February 2015. No legislative rule changes or requirements were associated with the endorsement. A survey link to assess use of the document was disseminated in August 2015.

Conclusions Results of the survey will be utilised to determine adoption of the document and inform future improvements and dissemination in Texas. Additionally, the Texas process has generated national attention and may be used to develop national standardised guidelines.

\section{COMMUNITY POLICE FOR SAFETY COMMUNITY}

Anon Namprasert. Khon Khen Province, Thailand

10.1136/injuryprev-2016-042156.619
Background Over alcohol drinking often caused altercation and nuisance in the community especially during festivals. Generally, community also encounter problems of drug addicted citizens who often tortured and injured people in the community which often caused violence loss of property and lives. This has been especially worried among the people in remote rural areas. This is because when there was violence in the area it was difficult for people to reach for helps from police in a timely manner. Therefore, a community police program was initiated.

Solving procedure A community police service unit has been established in the community in order to (1) support the community by setting community police in the community and (2) encourage people participation in protection measures (3) to strengthen the community to develop self-protected measures within the community

Working procedure 1. Call for community consensus for the development of police station in the area 2. If the location has been provided by the community, police department would instal necessary safety equipment in the unit 3 . Identify respectful and trustworthy citizen among the people in the community. These people will be trained to be a mediator for problem solving in the areas 4. Encourage citizen to join police volunteers. This volunteer should be able to participate in violence alleviation and protecting operation 5. Recruit citizen especially youth group that have a tendency to create nuisance in the community to be trained and assumed role as volunteer in guarding the community. 6. Allocate police officer to be supervisor and consultant to the unit of operation.

Results 1. The operation was implemented in Khon Kaen in 2013 where the project could establish 5 police operation units in each community with full supports from the people. 2 . There were 617 volunteers in 5 communities within these volunteers 66 people served as violence mediators, 136 police volunteers, 140 female volunteers for traffic accidents protection, 135 young police volunteers and 140 vulnerable youths who have been trained to serve as community guard and volunteers.

Outcome A significant drops in violence cases by $70.5 \%$, a decrease in drug related cases by $30 \%$, violence and nuisance caused by youth has reduced by $60 \%$, criminal cases dropped by $62.5 \%$ (statistics of police station at Kao Suan Kwang district, 2013) People in implemented community have been strengthen and empowered to become self- protected community.

Conclusion The development of community police unit together with strengthening systems should be an effective measures to create safe community in a sustainable procedures. The reduction in crime rate and violence in the community had been evident and the project has become an exemplary project for dissemination.

\section{0 DECREASE ROAD TRAFFIC INJURIES (RTI) MORTALITY IN UDONTHANI, THAILAND: 1997-2015}

${ }^{1}$ Daoruang Kommuangpuk, ${ }^{2}$ Anuchar Sethasathien, ${ }^{3}$ Jenjirustra Wongpratoom. ${ }^{1}$ Udonthani Hospital, Thailand; ${ }^{2}$ National Institute for Emergency Medicine, Thailand; ${ }^{3}$ Udonthani Public Health Provincial Office, Thailand

10.1136/injuryprev-2016-042156.620

The Global status report on road safety 2015, Thailand RTI mortality is the second in the world. Udonthani province is also confronted with this problem but dead case in 1997 to 2015 decrease from $816,573,494,374,424,484,474,373,382$, 330, 272, 274, 256, 302, 348, 293, 298, 309 and 247 (Budget 